\title{
Validité des deux espèces Onchocerca lienalis et O. gutturosa, chez les Bovins
}

\author{
par O. BAIN *, G. PETIT * et B. POULAIN * * \\ * Laboratoire de Zoologie (Vers) associé au C.N.R.S. \\ Muséum national d'Histoire naturelle, 43, rue Cuvier, F 75231 Paris Cedex 05 \\ ** Abattoirs de Versailles.
}

\section{Résuméé.}

Une nouvelle analyse morphologique de l'Onchocerque du ligament gastrosplénique fait apparaître des caractères morphologiques qui montrent qu'il s'agit d'une espèce valide, $O$. lienalis, distincte de l'Onchocerque cervicale, $O$. gutturosa.

Les microfilaires dermiques de ces 2 espèces ont des distributions totalement différentes : microfilaires d'O. gutturosa sont concentrées dans la région céphalique des Bovins, alors que celles d'O. lienalis se trouvent dans la région ombilicale. Nous soupçonnons donc fortement que le cycle évolutif qui s'effectue chez $S$. ornatum en Angleterre concerne, en fait, $O$. lienalis et non $O$. gutturosa, comme il était admis; il est plus vraisemblable qu'O. gutturosa évolue en réalité chez les Culicoïdes.

\section{Summary.}

Validity of the two species $O$. lienalis and $O$. gutturosa from Bovines.

A new morphological analysis of the Onchocerca from the gastrosplenic ligament reveals characteristics confirming the validity of the species $O$. lienalis as distinct from the cervical Onchocerca, $O$. gutturosa. The dermic microfilaire of the two species have completely different distributions: the microfilariae of $O$. gutturosa are gathered in the cephalic region of Bovines while that of $O$. lienalis can be found in the umbilical region. Therefore we suspect strongly that the life cycle evolving in $S$. ornatum in England is that of $O$. lienalis and nos of $O$. gutturosa as previously postulated; and we think that $O$. gutturosa is more likely to evolve in Culicoides than in Simulium.

Accepté le 25 janvier 1978. 
Les Bovins domestiques peuvent être parasités simultanément par une Onchocerque située dans le ligament gastrosplénique et une située au niveau du ligament cervical.

Johnston (1921) décrit la forme gastrosplénique comme une espèce distincte et reprend le taxon $O$. lienalis Stiles, 1892, pour la désigner. Cette espèce s'oppose à la forme cervicale, $O$. gutturosa Neumann, 1910 par trois principaux caractères: extrémité antérieure de la femelle et du mâle beaucoup plus fins, sans renflement cervical; cuticule de la femelle à côtes moins saillantes et intercôtes avec 2 stries au lieu de 4.

Par la suite, la plupart des auteurs adoptent une position différente, clairement exposée par Eichler (1973): les différences morphologiques entre ces deux formes sont très faibles et sont dues à la localisation différente des parasites; ces 2 espèces doivent donc être mises en synonymie (O. gutturosa, selon Eichler, 1973 et $O$. lienalis, selon Sonin, 1975).

Toutefois, encore actuellement, certains auteurs comme Beaver et coll. (1974) estiment que les caractères différentiels présentés par la cuticule des femelles suffisent pour distinguer les 2 espèces.

Le problème est donc de savoir quels sont, chez les Onchocerques, les caractères morphologiques qui ont une valeur spécifique sûre et si la cuticule de la femelle doit être rangée parmi ces caractères ?

Au cours des dernières années, un nombre important d'Onchocerques nouvelles a été étudié et les diagnoses d'espèces connues ont été précisées. Une méthode d'analyse morphologique plus complète a été mise au point, entraînant une meilleure compréhension de ce groupe de Filaires. Il est établi aujourd'hui que chaque espèce est définie par un grand nombre de caractères stables, dont les principaux sont: arrangement des papilles céphaliques et caudales, forme de l'œsophage et, chez la femelle, morphologie de la queue, de la microfilaire et de la cuticule. Si la valeur de ce dernier caractère a souvent été discutée, cela tient au fait que les meilleurs spécialistes ont eu parfois, pour faire leur analyse, un matériel fragmentaire ou mềme un mélange de 2 espèces [par exemple, Sandground, 1934, avec O. flexuosa (Wedl, (1856)]. Cela tient également au fait que la cuticule de la femelle n'a pas le même aspect d'une extrémité à l'autre du corps et qu'il faut donc effectuer les comparaisons entre les espèces sur des régions équivalentes du corps.

Nous avons donc repris l'étude de l'Onchocerque gastrosplénique des Bovins, sur du matériel récolté aux abattoirs de Versailles.

Chez la femelle, la cuticule de nos spécimens, à côtes basses avec 2 stries par intercôtes, correspond exactement à ce qui a été décrit dans d'autres régions du monde (Johnston, 1921, en Australie ; Beaver et coll. 1974, en Amérique du Nord). Fait nouveau, la microfilaire s'est révélée être distincte de celle d'O. gutturosa (pièces céphaliques plus puissantes, corps plus épais, etc.) et avoir également une répartition différente dans la peau des Bovins: des fragments de peau ombilicale et de peau frontale ont été prélevés aux abattoirs; les premiers contenaient exclusivement des microfilaires épaisses d'O. lienalis, les $2^{\circ}$ des microfilaires minces d'O. gutturosa. 
La cuticule de la femelle et la microfilaire, ainsi que d'autres caractères, permettent donc de distinguer clairement $O$. lienalis d'O. gutturosa. Les conséquences de cette dualité sont importantes pour la biologie de ces 2 espèces.

\section{I. - Redescription de la femelle et de la microfilaire d'Onchocerca lienalis}

Matériel : une demi-douzaine de ligaments gastrospléniques positifs, fixés à l'alcool $70^{\circ}$, ont permis de récolter, après digestion pepsique, une femelle entière, une région antérieure, deux régions postérieures et plusieurs fragments de corps de femelles, mais pas de mâle.

\section{Description de la femelle.}

Région antérieure extrêmement fine: $45 \mu$ de large à la hauteur de l'anneau nerveux, $50 \mu$ à la fin de l'œsophage, $85 \mu$ à $3 \mathrm{~cm}$ de la tête ; c'est seulement à $5 \mathrm{~cm}$ de la tête que le corps atteint à peu près sa largeur définitive $(180-220 \mu)$; la région postérieure est épaisse $(190 \mu)$ et plus enroulée que le reste du corps.

Le tégument dans son ensemble subit une forte torsion spiralée suivant l'axe longitudinal du corps (fig. $1 \mathrm{H}$ ).

Dans la région moyenne du corps, la cuticule, épaisse de 12 à $17 \mu$, est formée de 2 couches. La couche externe, qui est la moins épaisse, est ornée de côtes très étroites et peu saillantes; sur certains spécimens, elles sont bien visibles et ont un trajet très sinueux; sur la femelle entière, elles ne sont bien visibles qu'en coupe optique longitudinale sur les bords du corps; ces côtes sont espacées de 25 à $45 \mu$. La couche externe présente en outre un caractère original : sa surface est creusée de sillons longitudinaux, irrégulièrement distribués et particulièrement bien visibles sur coupe transversale ( $f$ ig. $2 A, B$ et $C$ ). La couche interne porte les stries transversales (2 par intercôtes); sur la femelle entière, dans certaines régions, les stries s'estompent, mais la face profonde de la couche interne présente à leur place des ondulations régulières.

A 1 centimètre de la tête, il n'y a qu'une couche cuticulaire et les côtes sont rectilignes et très fines.

Un demi-centimètre en avant de l'anus, la cuticule offre un aspect tout-à-fait comparable à celui de la région moyenne du corps, mais les côtes sont plus serrées (15 $\mu$ environ), plus saillantes, et les stries sont très marquées.

La cuticule n'est pas plus épaisse dans le plan latéral, sauf vers la région œsophagienne. Tout le long du corps, la musculature est épaisse et régulière ; les canaux excréteurs sont petits. 
La disposition des papilles de la tête est indiquée sur les figures $1 \mathrm{D}$, E et $\mathrm{F}$. Le pore excréteur est situé sous une légère saillie.
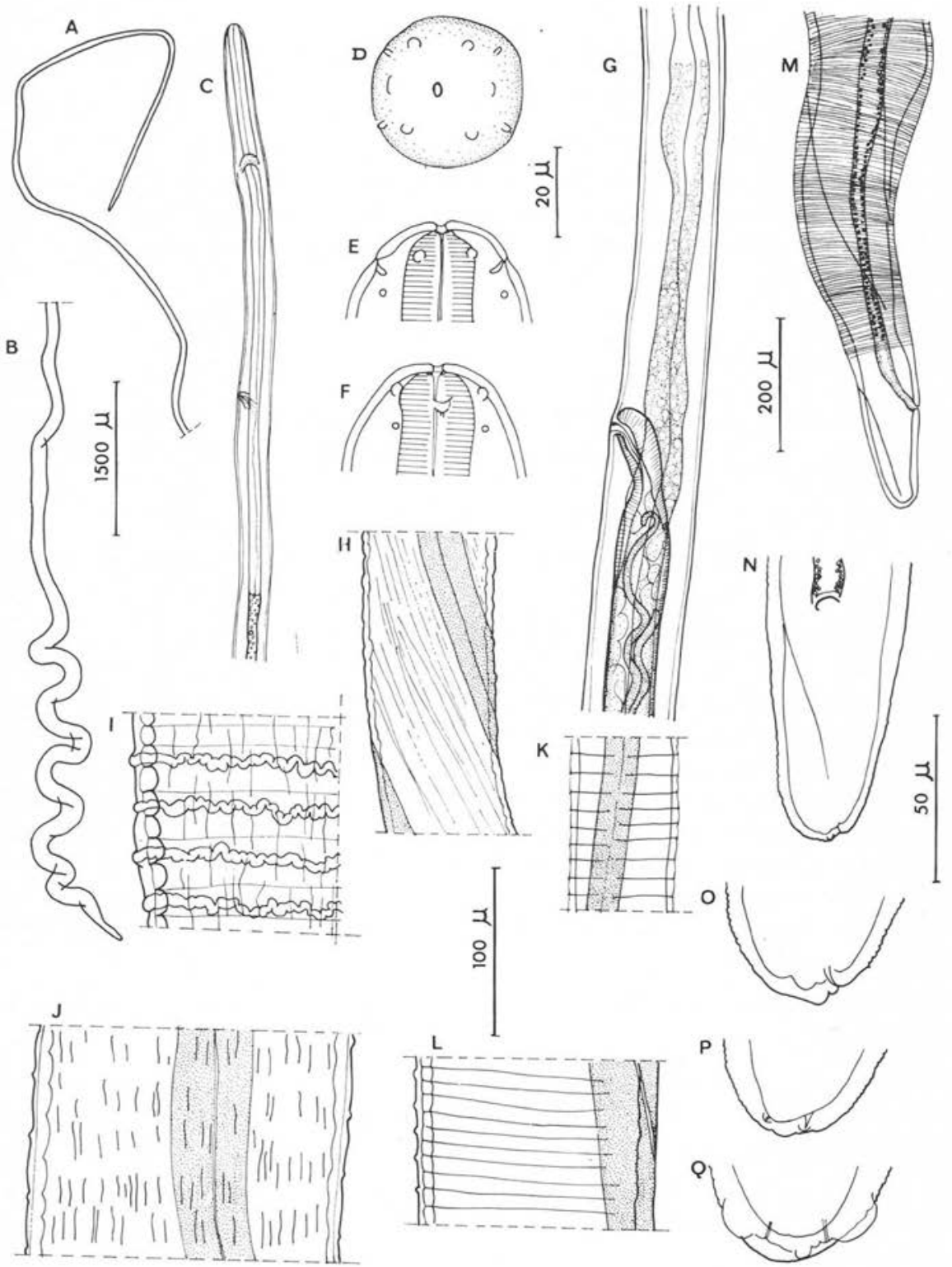
La vulve est située au niveau de la moitié postérieure de l'œsophage; celui-ci a une portion glandulaire à peine différenciée.

La queue est conique, à extrémité arrondie, les phasmides débouchent près de 2 mamelons dont la taille varie suivant les spécimens (fig.l $P, Q$ ) ; de profil, l'extrémité caudale apparaît divisée en un lobe ventral et un lobe dorsal plus développé; de face, le lobe ventral apparaît orné par un petit dôme surbaissé terminal.

- Femelle entière: Corps long de $316 \mathrm{~mm}$ et large au maximum de $220 \mu$; anneau nerveux, pore excréteur et vulve à 210,225 et $560 \mu$ de l'apex; œsophage musculaire et glandulaire longs de 340 et $525 \mu$; queue longue de $150 \mu$.

- Fragments de femelle: corps large de 180-220 $\mu$; anneau nerveux, pore excréteur et vulve à 210,240 et $543 \mu$ de l'apex, œsophage long de $1195 \mu$, à portion glandulaire de $770 \mu$; queue longue de $228 \mu$ et $193 \mu$.

\section{Description de la microfilaire.}

La microfilaire est relativement épaisse; la région antérieure a la même largeur que le corps; la forme de la tête est indiquée sur les figures $2 \mathrm{H}(1,2$ et 3$)$; le crochet est bien marqué; la queue est courte et se termine généralement par une pointe brusquement retrécie et courte.

En coloration vitale, la position des cellules excrétrice et $\mathrm{R}$ est indiquée sur la figure $2 \mathrm{G}$; la cellule $R_{1}$ est losangique.

7 microfilaires prélevées dans la région distale de l'ovéjecteur de femelles fixées à l'alcool à $70^{\circ}$, sont longues de 240 à $270 \mu(248,255,240,252,257,270$ et $258 \mu$ ) ; la largeur varie de 4,9 à $5,1 \mu$.

8 microfilaires provenant de peau ombilicale et colorées vitalement sont longues de 225 à $260 \mu(240,235,260,255,248,240,253$ et $225 \mu)$; la largeur varie de 5 à $5,3 \mu$. Pour une microfilaire longue de $253 \mu$, cellules excrétrices $R_{1}$ et $R_{4}$ à 104,180 et $217 \mu$. de l'apex.

Une microfilaire dermique, mesurée tuée en extension par la chaleur, est longue de $253 \mu$. et large de $5 \mu$.

Fig. 1. O. lienalis $\$$; A et $\mathrm{B}$ : silhouettes des régions antérieure et postérieure; C: région antérieure, vue latérale; D, E, F : tête en vues apicale, médiane et latérale; G: région vulvaire; $\mathrm{H}$ : région moyenne du corps, torsion spiralée du tégument; $\mathrm{I}$ et $\mathrm{J}$ : idem : aspects de la cuticule chez $2 \% ; \mathrm{K}$ : cuticule à $1 \mathrm{~cm}$ de la tête; $\mathrm{L}$ : cuticule à $1 \mathrm{~cm}$ de l'extrémité caudale; $\mathrm{M}$ : région caudale, vue latérale; $\mathrm{N}$ : queue, vue ventrale; $\mathrm{O}$ et $\mathrm{P}$ : extrémité caudale, vue latérale et ventrale; Q: idem, vue ventrale autre spécimen (éch.: A et B $1500 \mu$; C et M $200 \mu$; D, E, F $20 \mu$; O, P. Q $50 \mu$; G, H, I, J, K, L et N $100 \mu$ ). 

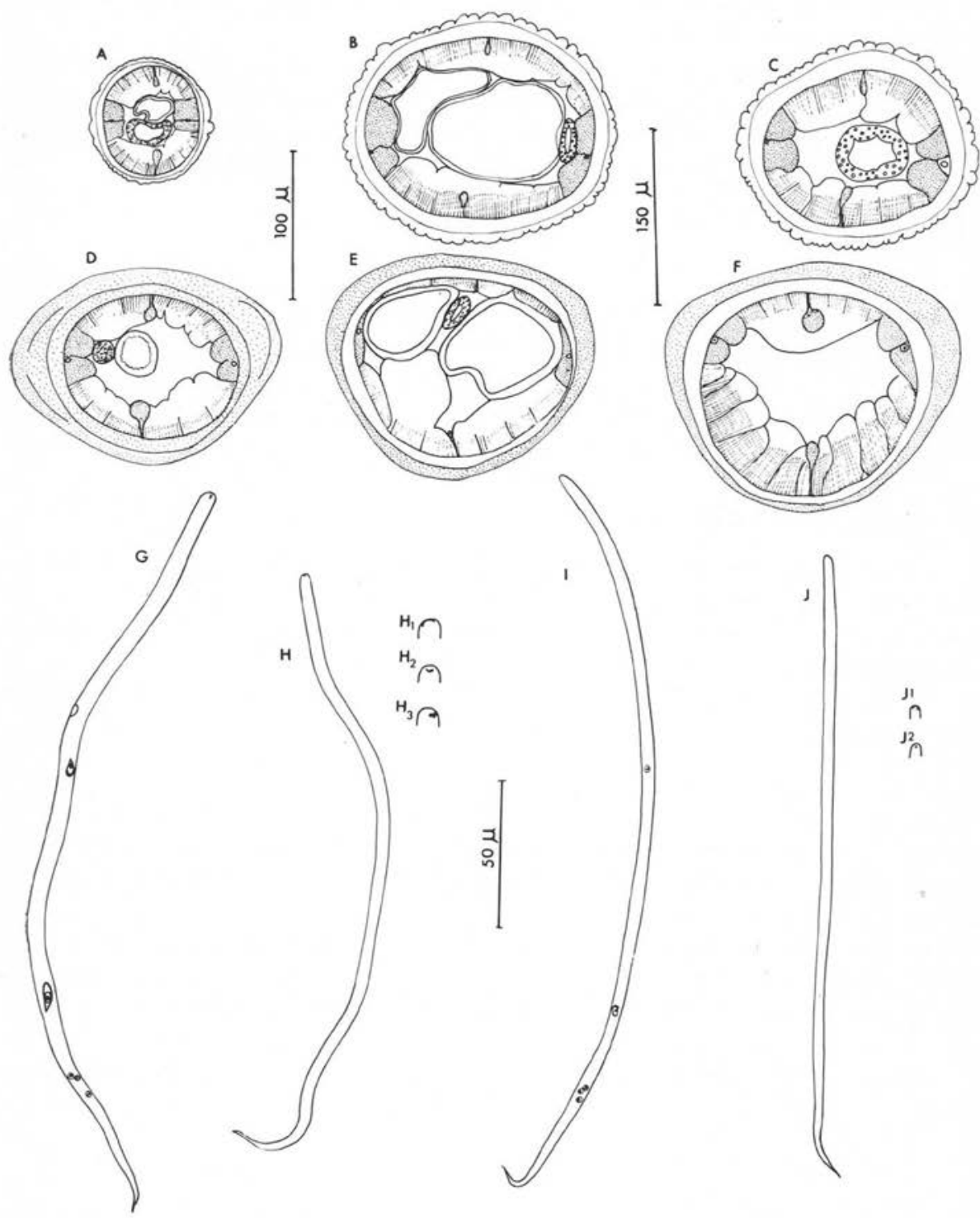

Fig. 2. Tégument chez la $q$ en coupes transversales effectuées à $2 \mathrm{~cm}$ de la tête, dans la région moyenne du corps et à $1 \mathrm{~cm}$ de l'extrémité caudale: $\mathrm{A}, \mathrm{B}, \mathrm{C}$ chez $O$. lienalis, D, E, F chez $O$. gutturosa. Microfilaires utérines et microfilaires dermiques en coloration vitale : $\mathrm{G}$ et $\mathrm{H}, O$. lienalis $\left(\mathrm{H}_{1}, \mathrm{H}_{2}, \mathrm{H}_{3}\right.$ tête, microfilaire à frais) I et $\mathrm{J}, O$. gutturosa $\left(\mathrm{J}_{1}, \mathrm{~J}_{2}\right.$ tête, microfilaire, fixée à l'alcool) (éch. : A, B, C, D et F $100 \mu ; \mathrm{E} 150 \mu ; \mathrm{G}, \mathrm{H}, \mathrm{I}, \mathrm{J}$ $50 \mu ; \mathrm{H}_{1}, \mathrm{H}_{2}, \mathrm{H}_{3}, \mathrm{~J}_{1}$ et $\mathrm{J}_{2}$ à main levée). 


\section{II. - Caractères différentiels entre $O$. lienalis et $O$. gutturosa}

Pour comparer l'Onchocerque cervicale à l'Onchocerque gastrosplénique, nous nous sommes servis d'une récente redescription (Bain, 1975), mais elle a dû être complétée par une étude plus précise du tégument (sur coupes transversales du corps de la femelle) et par un nouvelle étude de la microfilaire.

Le matériel analysé provient exclusivement d'Europe; il comprend, comme en 1975 , les lots $1255 \mathrm{JA}$ et $40 \mathrm{JA}$, utilisés pour l'étude de la femelle et des microfilaires utérines, et des microfilaires dermiques, observées à frais et récoltées dans des fragments de peaux frontales obtenues aux abattoirs de Versailles.

Les résultats de la comparaison des 2 Onchocerques sont les suivants :

\section{1. - Tégument (fig. $2 A$ à $F$ ) :}

Chez les 2 espèces, le tégument subit une torsion spiralée suivant l'axe longitudinal (cf. fig $7 \mathrm{G}$ in Bain, 1975), mais elle est plus accentuée chez O. lienalis.

Les autres différences sont nombreuses: chez $O$. gutturosa, les côtes sont très saillantes et il existe 4 stries par intercôte au lieu de 2 ; en outre, il existe chez cette espèce des «ailes » latéro-dorsales tout le long du corps, les sillons longitudinaux de la couche externe sont absents; enfin la musculature, beaucoup plus épaisse sur la face ventrale, les champs latéraux et les ailes déportés vers la face dorsale, montrent une asymétrie dorso-ventrale très forte qui n'existe pas chez $O$. lienalis.

\section{2. - Microfilaire (fig. $2 G$ à $J$ ) :}

$\mathrm{Si}$ on compare une préparation de microfilaires utérines mûres et bien fixées (1) d'O. lienalis à une préparation équivalente d'O. gutturosa, les différences apparaissent nettement : en effet, bien que les 2 sortes de microfilaires aient des longueurs sensiblement identiques (respectivement $240-270 \mu$ et $223-250 \mu$ sur les spécimens mesurés), leur silhouette diffère, car la microfilaire d' $O$. lienalis est large de 4,5 à $5 \mu$, alors que celle d'O. gutturosa n'atteint pas $4 \mu(3,5$ à $3,7 \mu)$.

De plus, la région antérieure d'O. lienalis a la même épaisseur que le corps et les pièces céphaliques sont bien visibles, tandis que, chez $O$. gutturosa, la région antérieure est généralement un peu amincie et les pièces céphaliques sont toujours très exiguës.

De telles différences se retrouvent chez les microfilaires de la peau des vaches: au niveau de l'ombilic, il existe une population homogène de microfilaires larges $(5$ à $5,3 \mu$ ) à pièces céphaliques relativement fortes et qu'il est donc facile d'assimiler à $O$. lienalis; au niveau du front il existe une deuxième population, presque homogène, constituée de microfilaires étroites $(3,7$ à $4 \mu$ en coloration vitale pour des longueurs

(1) Microfilaires extraites de l'ovéjecteur de femelles fixées à l'alcool chaud. 
de 240 à $270 \mu$ ) à pièces céphaliques très peu visibles et qui appartiennent donc à O. gutturosa.

\section{3. - Disposition des papilles de la tête :}

En vue apicale, chez O. gutturosa (fig. 7 in Bain, 1975), les papilles labiales externes et céphaliques apparaissent disposées sur un seul cercle, tandis que, chez $O$. lienalis, les papilles labiales externes apparaissent plus rapprochées de la bouche que les papilles céphaliques. La différence dans la position relative des papilles labiales et céphaliques apparaît aussi très clairement sur les vues latérales.

\section{4. - Autres caractères :}

Chez les 2 espèces, la queue porte un petit dôme terminal et 2 reliefs bordant les phasmides, mais ceux-ci sont arrondis chez $O$. lienalis et pointus chez $O$. gutturosa.

Enfin, la région antérieure est différente, extrêmement mince chez $O$. lienalis, puisqu'elle ne dépasse pas $45-50 \mu$ d'épaisseur; en outre, il n'existe pas chez cette espèce de renflement cervical, mais seulement une légère saillie ventrale correspondant au pore excréteur.

\section{III. - Conséquences \\ de la validité d'O. lienalis et $O$. gutturosa}

1. Plusieurs de nos descriptions concernant la microfilaire d'O. gutturosa son fausses:

- C'est le cas d'une microfilaire en coloration vitale, décrite par Bain (1971), et récoltée dans la peau ombilicale d'une vache en Angleterre; elle est large, à région antérieure aussi épaisse que le corps, et appartient manifestement à l'espèce $O$. lienalis.

- La figure $13 \mathrm{C}$ in Bain, 1975, représentant une microfilaire dermique ombilicale au Giemsa, récoltée en Camargue, doit aussi être interprétée comme $O$. lienalis.

Par contre, dans le même article, la figure $12 \mathrm{C}$ et $\mathrm{C}^{\prime}$ correspond véritablement à une microfilaire dermique d'O. gutturosa, récoltée chez une vache du Togo.

2. Les distributions des microfilaires dermiques d'O. gutturosa et $\mathrm{O}$. lienalis chez les Bovins ne sont pas identiques.

Venkataratnam et Kershaw (1961) ont tenté d'étudier la distribution des microfilaires dermiques en fonction de la localisation cervicale ou gastrosplénique des Onchocerques adultes; leurs observations aux abattoirs ont donné les résultats suivants :

- les microfilaires sont au niveau des oreilles et du cou chez les 3 Bovins où ont été trouvées uniquement des onchocerques cervicales: 
- elles sont au niveau du tronc, et non au niveau des oreilles, chez 11 des 16 animaux où ont été trouvées uniquement des onchocerques gastrospléniques ;

- elles sont dans les oreilles et le tronc de l'unique spécimen ayant une infestation mixte.

Ces observations sont conformes aux quelques sondages que nous avons faits aux abattoirs de Versailles: les microfilaires d'O. gutturosa n'ont été trouvées que dans la peau frontale, les microfilaires d'O. lienalis ont été trouvées dans la peau ombilicale et, en proportion très faible, dans la peau frontale.

$\mathrm{Au}$ Togo, où $O$. lienalis n'a jamais été trouvé chez les Bovins, les microfilaires d'O. gutturosa sont principalement concentrées à la base des cornes, au niveau des oreilles, du cou et du dos (Bain et coll., 1978). Fain et coll. en 1955 ont fait une constatation identique sur les Bovins du Ruanda Urundi parasités par $O$. gutturosa.

En Angleterre, la forte concentration en microfilaires au niveau de l'ombilic paraît donc être due, contrairement à ce qui était admis, non à $O$. gutturosa, mais à $O$. lienalis (cf. paragraphe III 1 sur l'identification morphologique des microfilaires ombilicales).

\section{Hypothèses sur les cycles d'O. lienalis et $\mathrm{O}$. gutturosa.}

Il est communément admis que le cycle d'O. gutturosa s'effectue chez les Simulies, dont l'espèce est différente suivant les régions.

En Angleterre, le vecteur reconnu est S. ornatum (voir Steward, 1937); cette Simulie se gorge sur la région ventrale des Bovins (Eichler, 1973), c'est-à-dire en fait, au niveau où se concentrent les microfilaires d'O. lienalis, et il est donc presque sûr que le cycle effectué soit celui d'O. lienalis.

A notre connaissance, la seule tentative pour effectuer les 2 cycles, celui d'O. gutturosa et celui d'O. lienalis, est due à Mikhailyuk (1967); selon cet auteur, les vecteurs sont, dans les 2 cas, des Simuliidæ, Boophtora sericata pour la première espèce, et $S$. galeratum pour la deuxième, mais aucune indication n'est donnée, ni sur le diagnostic et la localisation des microfilaires chez la vache, ni sur les lieux de piqûres de ces Simulies. Les expériences effectuées par l'auteur ne nous paraissent pas probantes, car les animaux en expérimentation étaient placés dans la nature sous moustiquaire et n'étaient donc pas à l'abri des piqûres de Culicoides.

Des observations effectuées au Togo nous suggèrent que le cycle d'O. gutturosa doit s'effectuer chez des Culicoides: en effet, les régions céphaliques et dorsales où se concentrent les microfilaires d'O. gutturosa ne sont pas piquées par des Simulies, mais par des Culicoides. Cette supposition avait été formulée par Le Roux dès 1947.

\section{Bibliographie}

Bain O. (1972): Recherches sur la morphogénèse des Filaires chez l'hôte intermédiaire. Ann. Parasitol. Hum. Comp., 47, 251-303.

Bain O. (1975): Redescription de cinq espèces d'Onchocerques. Ann. Parasitoi. Hum. Comp., 50, 763-788. 
Bain O., Denke A.-M., Amégée E., Chabaud A.-G. (1977) : Les Onchocerques des Bovins au Togo : les microfilaires et leur distribution. Ann. Univ. Benin, 3, 117-123.

Beaver P. C., Horner G. S., Bilos J. Z. (1974): Zoonotic onchocercosis in a resident of Illinois and observations on the identification of Onchocerca species. Ann. J. Trop. Med. Hyg., 23, 595607.

Eichler D. A. (1973): Studies on Onchocerca gutturosa (Neumann, 1910) and its development in Simulium ornatum (Meigen, 1818). 3. Factors affecting the development of the parasite in its vector. J. Helminthol., 47, 73-88.

Eichler D. A. (1973): Studies on Onchocerca gutturosa (Neumann, 1910) and its dévelopment in Simulium ornatum (Meigen, 1818). 4. Systematics of O. gutturosa. J. Helminthol. 47, 89-96.

Fain A., Herin V., Thienpont D. (1955) : Filiarioses des bovidés au Ruanda-Urundi. III. Etude parasitologique. B) Filaires des genres Setaria et Onchocerca et Microfilaires sanguines et dermiqués. Ann. Soc. Belge Méd. Trop., 35, 555-582.

Johnston T. H. (1921): Onchocerciasis of Queensland cattle. Trans. Proc. R. Soc. South Australia, 45, 231-247.

Le Roux P.L. (1947): Démonstration: 7. Proximal part of the $M$. biceps brachii from an Ox showing Onchocerca gutturosa infestation, Mazabuka, Northern Rhodesia. Trans. R. Soc. Trop. Med. Hyg., 41, 9.

Mikhailyuk A.P. (1967): O. gutturosa and $O$. lienalis in the wooded steppe zone of the Ukraine (en russe). Veterinariya, Kiev, 11, 62-67.

Sandground J.H. (1934): On the validity of the various species of the genus Onchocerca Diesing. Contrib. Dépt. Trop. Med. Inst. Trop. Biol. Med. (Harvard Univ.), 6, 133-172.

Sonin M.D. (1975): Osnovi Nematologii, Filariati, Onchocercini, 24, Acad. Sc. Edit., Moscou (en russe).

Steward J.S. (1937): The occurence of Onchocerca gutturosa Neumann in cattle in England, with an account of its life history and development in Simulium ornat:m Mg. Parasitology, 29, 212-219.

Venkataratnam A., Kershaw W. E. (1961): Distribution of the microfilariae of Onchocerca in the skin of cattle. Ann. Soc. Belge Med. Trop., 4, 323-328.

\section{ADDENDUM}

M. P. Gnedina (Dokladi Akad. Nauk SSSR), 70, 1950, 169-171) en Russie et R. Supperer (Wiener Tierärzt. Monastssch., 39, 1952, 173-179) en Autriche, ont attribué également à $O$. gutturosa les cycles qu'ils ont réalisés avec une Onchocerque du bétail chez Odagmia ornata. Tous deux admettent la synonymie des deux Onchocerques; l'excellente description de la microfilaire donnée par Supperer correspond à $O$. lienalis. 